\title{
Systematic understanding of acute effects of intravenous guanfacine on rat carotid sinus baroreflex-mediated sympathetic arterial pressure regulation
}

Toru Kawada ${ }^{\mathrm{a}}$, Shuji Shimizu ${ }^{\mathrm{a}}$, Michael J Turner ${ }^{\mathrm{a}}$, Masafumi Fukumitsu ${ }^{\mathrm{a}}$, Hiromi Yamamoto ${ }^{\mathrm{b}}$, Masaru Sugimachi ${ }^{\mathrm{a}}$

${ }^{a}$ Department of Cardiovascular Dynamics, National Cerebral and Cardiovascular Center, Osaka 565-8565, Japan. ${ }^{\mathrm{b}}$ Division of Cardiology, Department of Medicine, Faculty of Medicine, Kinki University, Osaka 589-8511, Japan

\section{Correspondence:}

Toru Kawada, MD, PhD

Department of Cardiovascular Dynamics

National Cerebral and Cardiovascular Center

Osaka 565-8565, Japan

Phone: $\quad+81-6-6833-5012$

Fax: $\quad+81-6-6835-5403$

E-mail:_torukawa@ncvc.go.jp 


\section{Abstract}

Aims: To assess the acute effects of intravenous guanfacine, an $\alpha_{2 A}$-adrenergic agonist, on sympathetic outflow from the central nervous system and on sympathetic arterial pressure (AP) response.

Main methods: In anesthetized Wistar Kyoto rats, carotid sinus baroreceptor regions were isolated. Changes in electrical sympathetic nerve activity (SNA) and AP in response to a baroreceptor pressure input were examined before and after an intravenous administration of a high dose $(100 \mu \mathrm{g} / \mathrm{kg}, \mathrm{n}=7)$ or a low dose $(20 \mu \mathrm{g} / \mathrm{kg}$, $\mathrm{n}=5$ ) of guanfacine.

Key findings: The higher dose of guanfacine significantly narrowed the range of the AP response $(86.8 \pm 6.4$ to $38.4 \pm 12.9 \mathrm{mmHg}, \mathrm{P}<0.01)$ but increased the minimum AP $(79.3 \pm 7.5$ to $93.2 \pm 8.7 \mathrm{mmHg}, \mathrm{P}<0.05)$. In the neural arc, guanfacine reduced both the response range $(90.4 \pm 2.3$ to $33.4 \pm 10.7 \%, \mathrm{P}<0.01)$ and the minimum SNA $(11.4 \pm 1.9$ to $2.6 \pm 1.5 \%, P<0.01)$. In the peripheral arc, guanfacine increased the intercept $(67.6 \pm 7.1$ to $92.8 \pm 8.5 \mathrm{mmHg}, \mathrm{P}<0.01)$ without a significant effect on the slope. The lower dose of guanfacine weakened the effects on both the neural and peripheral arcs.

Significance: Guanfacine suppressed SNA without a significant reduction of AP, which may be attributable to the peripheral vasoconstrictive effect. Reducing the dose of acutely administered intravenous guanfacine does not aid in separating the central sympathoinhibitory effect from the peripheral vasoconstrictive effect on AP in anesthetized rats in vivo.

Keywords: Guanfacine, Carotid sinus baroreflex, Open-loop systems analysis, Sympathetic nerve activity, Alpha-2 agonist 


\section{Introduction}

Guanfacine, an $\alpha_{2 A}$-adrergic agonist, exerts an antihypertensive effect by suppressing electrical sympathetic nerve activity (SNA) via a mechanism of presynaptic inhibition of adrenergic neurons in the central nervous system [1-3]. Guanfacine can also function peripherally to inhibit norepinephrine release in response to adrenergic nerve stimulation in an isolated rabbit heart preparation [4]. On the other hand, a previous study from our laboratory indicated that intravenous guanfacine increased vagal acetylcholine release with little effect on sympathetic norepinephrine release in the atrial myocardial interstitium in anesthetized rabbits [5]. In the same study, intravenous guanfacine decreased heart rate (HR) but did not significantly affect arterial pressure (AP).

Several factors need to be considered to reconcile the above-mentioned diverse experimental results. First, background sympathetic tone was not intentionally increased in our previous study [5], which might have made it difficult to demonstrate sympathoinhibitory and hypotensive effects of guanfacine. However, this limitation is not always evident because intravenous medetomidine, another $\alpha_{2}$-adrenergic agonist, reduced myocardial norepinephrine release and decreased AP in the same experimental setting [6]. Medetomidine is different from guanfacine in that it may induce a hypotensive effect via imidazoline receptors because medetomidine is an imidazole [7]. Second, while peripheral vasoconstriction is predominantly mediated by postsynaptic $\alpha_{1}$-adrenrgic receptors, postsynaptic $\alpha_{2}$-adrenergic receptors also contribute to vasoconstriction in several vascular beds [8]. Guanfacine can induce pressor responses in pithed rats by acting on $\alpha_{2}$-adrenergic receptors at low doses (in the range less than or equal to $10 \mu \mathrm{g} / \mathrm{kg}$ ) and by additionally stimulating $\alpha_{1}$-adrenrgic 
receptors at high doses (in the range greater than or equal to $30 \mu \mathrm{g} / \mathrm{kg}$ ) [9]. These peripheral vasoconstrictive effects might have counteracted the centrally-mediated hypotensive effect of guanfacine. Finally, under baroreflex closed-loop conditions, changes in SNA and AP by a given drug are attenuated by a negative-feedback mechanism. For instance, if AP is decreased via a central sympathoinhibitory effect of guanfacine, the decrease in AP acts to increase SNA via the arterial baroreflex, counteracting any sympathoinhibitory effect.

In order to more systematically understand the total picture of the effects of guanfacine on SNA and AP, this study employed a framework of open-loop analysis of the carotid sinus baroreflex where the carotid sinus baroreceptor regions were isolated from systemic circulation [10-14]. In this method, the baroreceptor input pressure is controlled externally independent of changes in AP, which enables the examination of the effect of intravenous guanfacine at different levels of SNA. Furthermore, this method enables the separate estimation of the characteristics of the two principal arcs of the arterial baroreflex system, i.e., the neural and peripheral arcs $[15,16]$. The neural arc represents the input-output relationship between baroreceptor input pressure and SNA, whereas the peripheral arc represents the input-output relationship between SNA and AP. The central effect through presynaptic $\alpha_{2}$-adrenergic receptors has been the major focus for the usage of guanfacine because a vasoconstrictive effect via postsynaptic $\alpha_{2}$-adrenergic receptors may be regarded as secondary compared with that via postsynaptic $\alpha_{1}$-adrenergic receptors [17]. We hypothesized, however, that the peripheral vasoconstrictor effect of guanfacine may be stronger than generally understood and cannot be ignored in the determination of AP during the acute intravenous administration of guanfacine. 


\section{Materials and methods}

Surgical Preparation

The animal experiments in this study were carried out in accordance with the National Institutes of Health guide for the care and use of Laboratory animals (NIH Publications No. 8023, revised 1978). The experimental protocols were reviewed and approved by the Animal Subject Committee of the National Cerebral and Cardiovascular Center. Twelve male Wistar Kyoto (WKY) rats (394 $\pm 29 \mathrm{~g}$, mean \pm SD) were artificially ventilated after inducing anesthesia with a peritoneal injection of a mixture of urethane $(250 \mathrm{mg} / \mathrm{ml})$ and $\alpha$-chloralose $(40 \mathrm{mg} / \mathrm{ml})$ at a dose of $2 \mathrm{ml} / \mathrm{kg}$. To maintain a suitable level of anesthesia, the anesthetic mixture was diluted by physiological saline to a one-eighteenth concentration and continuously administered at a rate of $1 \mathrm{ml} / \mathrm{kg}$ through a venous catheter inserted into the right femoral vein. Another venous catheter inserted into the left femoral vein was used to administer test drugs. An arterial catheter was introduced from the right femoral artery to measure AP and HR. A pair of stainless steel wire electrodes were attached to a postganglionic branch of the splanchnic sympathetic nerve to record SNA $[10,13]$. Briefly, a biosignal amplifier (AB-610J, Nihon Kohden, Japan) magnified the nerve activity by 200,000 times (i.e., $1 \mathrm{~V} / 5 \mu \mathrm{V}$ ) with a bandpass filter between 150 and 1000 $\mathrm{Hz}$. The signal was then fed into an analog circuit for full-wave rectification and low-pass filtering with a cut-off frequency of $30 \mathrm{~Hz}$ to quantify SNA. Bilateral carotid sinus baroreceptor regions were isolated $[18,19]$ to control carotid sinus pressure (CSP). Bilateral vagal and aortic depressor nerves were sectioned to minimize any confounding effects through reflexes from the cardiopulmonary region and aortic arch. Open-loop static characteristics of the carotid sinus baroreflex were estimated as 
follows. CSP was first decreased to $60 \mathrm{mmHg}$ for $5 \mathrm{~min}$, then increased in a staircase manner from 60 to $180 \mathrm{mmHg}$ in increment of $20 \mathrm{mmHg}$. Each step was maintained for $60 \mathrm{~s}$. Carotid sinus baroreceptors were exposed to non-pulsatile pressure [10-14]. Protocols

In Protocol 1 (high dose, $\mathrm{n}=7$ ), guanfacine was intravenously administered as a bolus $(100 \mu \mathrm{g} / \mathrm{kg})$. Guanfacine was dissolved in physiological saline at a concentration of $100 \mu \mathrm{g} / \mathrm{ml}$, and the solution was injected at a dose of $1 \mathrm{ml} / \mathrm{kg}$ over a duration of approximately $10 \mathrm{~s}$. In our previous study, this dose of guanfacine effectively increased cardiac vagal acetylcholine release with little effect on sympathetic norepinephrine release in the rabbit atrial myocardium [5]. This dose was above the dose (approximately $30 \mu \mathrm{g} / \mathrm{kg}$ ) that caused a $50 \%$ increase in AP relative to the maximum response in pithed rats [9]. Twenty minutes later, when hemodynamic changes reached a new steady state, the baroreflex responses were compared with those acquired before administering guanfacine. The effect of an injected volume (1 $\mathrm{ml} / \mathrm{kg}$ ) was assumed to be minimal as we waited for 20 min after guanfacine administration to analyze the data. The control data were acquired without a vehicle (physiological saline) pretreatment. At the end of the protocol, hexamethonium bromide was intravenously administered at a dose of $60 \mathrm{mg} / \mathrm{kg}$ to block the sympathetic ganglionic transmission and record the noise level of SNA.

In Protocol 2 (low dose, $n=5$ ), the dose of guanfacine was reduced to 20 $\mu \mathrm{g} / \mathrm{kg}$ and the effect on baroreflex responses was examined. Guanfacine was dissolved in physiological saline at a concentration of $20 \mu \mathrm{g} / \mathrm{ml}$, and the solution was injected at a dose of $1 \mathrm{ml} / \mathrm{kg}$ over a duration of approximately $10 \mathrm{~s}$. This dose was below the dose (approximately $30 \mu \mathrm{g} / \mathrm{kg}$ ) that caused a $50 \%$ increase in AP relative to 
the maximum response in pithed rats [9]. This protocol was added based on the results of Protocol 1 to test the hypothesis that the lower dose could be effective in avoiding a peripheral vasoconstrictive effect while maintaining the central sympathoinhibitory effect of guanfacine (see Discussion).

\section{Data Analysis}

The staircase-wise input cycles are referred to as S1 through S7 (Fig. 1). Guanfacine was administered as a bolus $1 \mathrm{~min}$ after the completion of S2. The baroreflex responses obtained from the S2 cycle were treated as control, and those obtained from the S5 cycle were used to evaluate the effects of guanfacine. In each input cycle, data averaged for the last $10 \mathrm{~s}$ at each CSP level were used to quantify static characteristics of the carotid sinus baroreflex. SNA was normalized in each animal so that the SNA value at a CSP of $60 \mathrm{mmHg}$ under the control condition became $100 \%$ and that after ganglionic blockade became $0 \%$. The input-output data for the relationships between CSP and AP (total reflex arc), between CSP and SNA (neural arc), and between CSP and HR were analyzed using a four-parameter logistic function, and the response range $\left(P_{1}\right)$, slope coefficient $\left(P_{2}\right)$, midpoint input pressure $\left(P_{3}\right)$, and minimum value $\left(P_{4}\right)$ were estimated $[12,20]$. As for the relationship between SNA and AP (peripheral arc), a linear regression analysis was used to obtain the intercept and slope of the relationship [12]. The operating point of the carotid sinus baroreflex was calculated from a baroreflex equilibrium diagram [12, 16, 21].

Statistical Analysis

Data are presented as means \pm standard error. Fitted parameter values were compared before and after administering guanfacine using a paired t-test in each protocol [22]. The differences were considered statistically significant when $P<0.05$. 


\section{Results}

Shown in Figures 1 are the representative time series of the high dose protocol. Gray lines in the AP and HR panels indicate $200-\mathrm{Hz}$ resampled data. Gray lines in the SNA panels indicate $10-\mathrm{Hz}$ resampled data. Black lines in AP, SNA, and HR panels indicate 2-s moving averaged data. Before administering guanfacine, an increase in CSP decreased AP, SNA, and $H R$, reflecting the negative feedback operation of the carotid sinus baroreflex. Guanfacine gradually decreased the maximum AP, but it increased the minimum AP at the same time. Guanfacine acutely suppressed SNA, and the suppression persisted until the end of the protocol. The maximum HR gradually decreased after guanfacine. In contrast to the increase in the minimum AP, the minimum HR tended to decrease after guanfacine.

Shown in Figures 2 are the representative time series of the low dose protocol. Guanfacine slightly decreased the maximum AP with little change in the minimum AP. The low dose of guanfacine suppressed SNA, but the effect was weaker than that of the high dose. There was a notch-like HR response during S1 and S2 in this animal. The notch was created by a gradual increase in HR even though SNA was suppressed (middle panels). The phenomenon was not commonly observed across animals and is likely peculiar to this animal. The maximum HR decreased, and the minimum HR tended to decrease after guanfacine.

The open-loop static characteristics of the carotid sinus baroreflex are compared between the S2 and S5 cycles in Figures 1 and 2. Group-averaged data are summarized in Figure 3 and Table 1. The effects of the high dose of guanfacine are-depicted in Figure 3A through 3D. In the total reflex arc (Fig. 3A), the range of AP 
response $\left(P_{1}\right)$ was significantly narrowed whereas the minimum AP $\left(P_{4}\right)$ was increased by guanfacine. The maximum gain of the total reflex arc was reduced by guanfacine. In the HR regulation (Fig. 3B) and the neural arc (Fig. 3C), the response range $\left(P_{1}\right)$ was narrowed and the minimum value $\left(P_{4}\right)$ was reduced by guanfacine. The maximum slope in the HR regulation and the neural arc were also reduced by guanfacine. In the peripheral arc (Fig. 3D), the intercept was significantly increased, but the slope was not significantly changed by guanfacine.

The effects of the low dose of guanfacine are illustrated in Figure 3E through $3 \mathrm{H}$. The total reflex arc (Fig. 3E) was not affected much by guanfacine, though the decrease in the minimum $\mathrm{AP}\left(P_{4}\right)$ was statistically significant. In the HR regulation (Fig. 3F), the range of HR response $\left(P_{1}\right)$ was narrowed. In the neural arc (Fig. 3G), the minimum SNA $\left(P_{4}\right)$ was reduced, but the other parameters were not changed significantly by guanfacine. In the peripheral arc (Fig. $3 \mathrm{H}$ ), the intercept was significantly increased by the low dose of guanfacine, but the effect was smaller than that induced by the high dose. The slope was not significantly affected by the low dose of guanfacine.

Baroreflex equilibrium diagrams constructed using mean parameter values before and after administering guanfacine are shown in Figure 4. The intersection between the neural and peripheral arcs yields the operating point at which SNA and AP are settled under baroreflex closed-loop conditions. In the high dose protocol (Fig. 4A), guanfacine moved the neural arc leftward (i.e., toward lower SNA). If guanfacine had not affected the peripheral arc, the operating point might have changed from the open circle to the open triangle, resulting in significant reduction of the operating-point AP. However, because guanfacine moved the peripheral arc upward (i.e., toward 
higher AP), the operating-point AP did not decrease significantly despite the significant reduction of the operating-point SNA (the filled circle). The operating-point gain was significantly reduced by the high dose of guanfacine (Table 1). In the low dose protocol (Fig. 4B), the effects of guanfacine on the neural and peripheral arcs were both weaker compared with the high dose. While the operating-point SNA was reduced by guanfacine, the operating-point AP was not significantly changed (the open circle vs. the gray circle). The operating-point gain was not significantly affected by the low dose of guanfacine (Table 1).

\section{Discussion}

We have shown that the intravenous administration of a high dose of guanfacine $(100 \mu \mathrm{g} / \mathrm{kg})$ significantly reduced SNA at any given CSP, and the effect was greater at low CSP levels (Fig. 3C). On the other hand, guanfacine increased AP at any given level of SNA, and the effect seemed to be independent of SNA levels (Fig. 3D). This latter vasoconstrictive effect seems to be less highlighted when guanfacine is used as a centrally-acting agent [17], but the vasoconstriction occurs regardless of the SNA levels, which contributes to the determination of AP in vivo. Guanfacine decreased AP at low CSP levels (i.e., at high SNA levels) whereas increased AP at high CSP levels (i.e., at low SNA levels) (Fig. 3A). When the dose was reduced to $20 \mu \mathrm{g} / \mathrm{kg}$, the effects of guanfacine on both the neural and peripheral arcs were attenuated.

Effect of intravenous guanfacine on the baroreflex neural arc

Pharmacological agents that have $\alpha_{2}$-adrenergic agonist activity such as clonidine are known to inhibit SNA via $\alpha_{2 A}$-adrenergic receptors as a class effect [17, 
23]. Guanfacine is a selective $\alpha_{2 A}$-adrenergic agonist, and at low doses (e.g., less than or equal to $50 \mu \mathrm{g} / \mathrm{kg}$ ), it can exert a neuropsychiatric effect without inducing significant hypotension and sedation [24]. As such, guanfacine is now used to treat attention deficit hyperactive disorder in children and young adolescents [25]. In our previous study in anesthetized rabbits, intravenous guanfacine at a dose of $100 \mu \mathrm{g} / \mathrm{kg}$ decreased HR without significantly reducing AP [5]. In that study, acetylcholine release was significantly increased but norepinephrine release was not significantly changed in the atrial myocardial interstitium. In contrast, medetomidine, another $\alpha_{2}$-adrenergic agonist, significantly increased acetylcholine release with a significant decrease in norepinephrine release [6]. Although clonidine and medetomidine bind to imidazoline receptors as well as $\alpha_{2}$-adrenergic receptors [7], guanfacine does not have an imidazoline structure. While the contribution of imidazoline receptors to the hypotensive effect during systemic administration of clonidine has been argued in anesthetized rats [26], we speculated that the lack of imidazoline structure could be a possible reason for the relatively weak sympathoinhibitory effect of guanfacine in our previous study [5].

In the present study, SNA was significantly reduced by the high dose of guanfacine (Fig. 3C). The fact that the attenuation was more evident at low CSP levels indicates that guanfacine is more effective in reducing high levels of SNA. Hence, another factor for the little effect of guanfacine on norepinephrine release in our previous study [5] is that SNA was not activated enough to uncover the sympathoinhibitory effect of guanfacine. A species difference between rabbits and rats may also need to be considered to reconcile the discrepancy. While the vagal nerves were sectioned, the high dose of guanfacine significantly reduced the range of 
the HR response (Fig. 3B), suggesting that cardiac SNA might have been suppressed parallel with splanchnic SNA. Reducing the dose of guanfacine to $20 \mu \mathrm{g} / \mathrm{kg}$ weakened the sympathoinhibitory and bradycardic effects (Fig. 3G and 3F).

Effect of intravenous guanfacine on the baroreflex peripheral arc

In the isolated rabbit heart, guanfacine inhibits norepinephrine release in response to adrenergic nerve stimulation [4]. If norepinephrine release from sympathetic nerve terminals was also significantly attenuated by the activation of presynaptic $\alpha_{2}$-adrenergic receptors in the vasculature, sympathetic AP response might have been attenuated. In the present study, however, the intercept of the regression line between SNA and AP was significantly increased by guanfacine (Fig. 3D). While a vasoconstrictive effect is mainly mediated by postsynaptic $\alpha_{1}$-adrenergic receptors, postsynaptic $\alpha_{2}$-adrenergic receptors also contribute to the vasoconstriction $[8,27]$. In tail arteries of WKY, postsynaptic $\alpha_{2}$-adrenergic receptors are present and play a role in vasoconstriction [28]. It is likely that the vasoconstrictive effect of guanfacine mediated by postsynaptic $\alpha_{2}$-adrenergic stimulation overcame the inhibition of peripheral norepinephrine release mediated by presynaptic $\alpha_{2}$-adrenergic stimulation in the vasculature, and caused a net increase in AP at any given SNA. The duration of vasoconstrictive effect may differ among $\alpha_{2}$-adrenergic agonists, because intravenous medetomidine reduces AP within approximately 10 min following a short hypertensive response [29].

In previous studies under baroreflex closed-loop conditions, it seems difficult to quantify the relative potency of the AP increase via the peripheral vasoconstrictive effect compared with the AP decrease via the central sympathoinhibitory effect. The high dose protocol clearly demonstrated that the central sympathoinhibitory effect 
overcame the vasoconstrictive effect at low CSP levels (at high SNA levels), resulting in the manifestation of a hypotensive effect (Fig. 3A). In contrast, the vasoconstrictive effect overcame the central sympathoinhibitory effect at high CSP levels (at low SNA levels), resulting in the manifestation of a hypertensive effect. Because of these opposing scenarios, the operating-point AP was not changed significantly by the high dose of guanfacine (Fig. 4A). For the HR response, because the sympathetic HR response depends chiefly on $\beta$-adrenergic receptors, only the bradycardic effect associated with the central sympathoinhibitory effect was observed regardless of the SNA levels (Fig. 3B). For the HR response, peripheral inhibition of norepinephrine release from the sympathetic nerve terminals via the activation of presynaptic $\alpha_{2}$-adrenergic receptors might have also contributed to the bradycardic effect [4]. Implication of the study

An autonomic imbalance with an excess SNA and reduced parasympathetic nerve activity is considered to aggravate cardiovascular diseases such as chronic heart failure. Sympathetic suppression by $\beta$-blockers [30], angiotensin converting enzyme inhibitors [31], and angiotensin II receptor blockers [32] is a potent therapy for heart failure. Peak decreases in mean AP, HR, and renal SNA in response to intracerebroventricular guanabenz are augmented in chronic heart failure, which can be treated by chronic intracerebroventricular losartan [33]. The study demonstrated an involvement of central $\alpha_{2}$-adrenergic mechanism in the sympathoexcitation observed in chronic heart failure. Pharmacological and electrical vagal activation can improve the survival of rats with chronic heart failure after myocardial infarction [3436]. Previous studies from our laboratory indicated that $\alpha_{2}$-adrenergic stimulation by intravenous guanfacine or medetomidine can increase vagal acetylcholine release in 
rabbits and rats $[5,6,29,37]$. The feature of central sympathoinhibition and vagal activation of these drugs seems to be ideal for the treatment of the autonomic imbalance associated with cardiovascular diseases. Medetomidine, however, has a strong sedative effect, which may hamper its application to outpatients. On the other hand, guanfacine, at low doses, can exert a central effect without inducing hypotension and sedation [25]. While a central sympathoinhibitory effect was confirmed in the present study, the peripheral vasoconstrictive effect was not negligible and may antagonize the treatment purpose of afterload reduction in chronic heart failure. Therefore, guanfacine may not be ideal for the use as a treatment of chronic heart failure to achieve central sympathoinhibition and vagal activation. We predicted that the peripheral vasoconstrictive effect could be avoided by decreasing the dose of guanfacine in Protocol 2, however, the central sympathoinhibitory effect was also reduced parallel to the vasoconstrictive effect, at least, in the present experiment (Fig. $3 \mathrm{G}$ and $3 \mathrm{H}$ ).

\section{Limitations}

The present results cannot account for the chronic effects under conscious conditions because guanfacine was administered only acutely under anesthetic conditions. In addition, there are significant regional and species differences in the relative contribution of $\alpha_{1}$ - and $\alpha_{2}$-adrenergic receptors to vasoconstriction [8, 27]. While the present study suggested that the central sympathoinhibitory effect of guanfacine is not easily separable from the peripheral vasoconstrictive effect, the results could be different in other species and strains. The WKY rat is known as a model of depression [38]. While the present study was performed under anesthetic conditions and behavioral characteristics were not the focus of the study, a possibility 
cannot be ruled out that the depressive nature of WKY may have affected the baroreflex-mediated SNA response. Furthermore, the present study does not include a control group without baroreceptor isolation and without vagal and aortic nerve section. Hence, the results may not be directly extrapolated to the intact condition where baroreceptors are exposed to pulsatile pressure and interactions between multiple reflex systems exist in the regulation of SNA and AP.

\section{Conclusions}

Guanfacine suppressed SNA without a significant reduction in AP, which may be attributable to a peripheral vasoconstrictive effect. Reducing the dose of acutely administered intravenous guanfacine does not aid in separating the central sympathoinhibitory effect from the peripheral vasoconstrictive effect on AP in anesthetized rats in vivo.

\section{Acknowledgements}

This study was partly supported by a Grant-in-Aid for Scientific Research (25350909, $26750153,15 \mathrm{~K} 09110,15 \mathrm{H} 03101)$ and the support program to break the bottlenecks at R\&D System for accelerating the practical use of the Health Research Outcome from the Japan Science and Technology Agency. 


\section{References}

[1] P. MacCarthy, P. Isaac, G. Frost, A. Freeman, G. Stokes, Clinical dose-response studies with guanfacine (BS 100-141), a new antihypertensive agent, Clin. Exper. Pharmacol. Physiol. 5 (1978) 187-190.

[2] S.Z. Langer, N. Duval, R. Massingham, Pharmacologic and therapeutic significance of $\alpha$-adrenoceptor subtypes, J. Cardiovasc. Pharmacol. 7(Suppl 8) (1985) S1-S8.

[3] G. Scholtysik, Animal pharmacology of guanfacine, Am. J. Cardiol. 57 (1986) 13E-17E.

[4] W. Pacha, R. Salzmann, G. Scholtysik, Inhibitory effects of clonidine and BS 100-141 on responses to sympathetic nerve stimulation in cats and rabbits, $\mathrm{Br}$. J. Pharmacol. 53 (1975) 513-516.

[5] S. Shimizu, T. Kawada, T. Akiyama, M.J. Turner, T. Shishido, A. Kamiya, M. Shirai, M. Sugimachi, Guanfacine enhances cardiac acetylcholine release with little effect on norepinephrine release in anesthetized rabbits, Auton. Neurosci. 187 (2015) 84-87.

[6] S. Shimizu, T. Akiyama, T. Kawada, A. Kamiya, M.J. Turner, H. Yamamoto, T. Shishido, M. Shirai, M. Sugimachi, Medetomidine suppresses cardiac and gastric sympathetic nerve activities but selectively activates cardiac vagus nerve, Circ. J. 78 (2014) 1405-1413.

[7] Z.P. Khan, C.N. Ferguson, R.M. Jones, Alpha-2 and imidazoline receptor agonists. Their pharmacology and therapeutic role, Anaesthesia 54 (1999) 146165.

[8] S.Z. Langer, P.E. Hicks, Alpha-adrenoceptor subtypes in blood vessels: physiology and pharmacology, J. Cardiovasc. Pharmacol. 6(Suppl 4) (1984) 
S547-S558.

[9] P.B. Timmermans, H.Y. Kwa, P.A. van Zwieten, Possible subdivision of postsynaptic $\alpha$-adrenoceptors mediating pressor responses in the pithed rat, Naunyn-Schmiedeberg's Arch. Pharmacol. 310 (1979) 189-193.

[10] T. Kawada, A. Kamiya, M. Li, S. Shimizu, K. Uemura, H. Yamamoto, M. Sugimachi, High levels of circulating angiotensin II shift the open-loop baroreflex control of splanchnic sympathetic nerve activity, heart rate and arterial pressure in anesthetized rats, J. Physiol. Sci. 59 (2009) 447-455.

[11] T. Kawada, M. Li, A. Kamiya, S. Shimizu, K. Uemura, H. Yamamoto, M. Sugimachi, Open-loop dynamic and static characteristics of the carotid sinus baroreflex in rats with chronic heart failure after myocardial infarction, J. Physiol. Sci. 60 (2010) 283-298.

[12] T. Kawada, M. Sugimachi, Open-loop static and dynamic characteristics of the arterial baroreflex system in rabbits and rats, J. Physiol. Sci. 66 (2015) 15-41.

[13] H. Yamamoto, T. Kawada, S. Shimizu, A. Kamiya, S. Miyazaki, M. Sugimachi, Effects of cilnidipine on sympathetic outflow and sympathetic arterial pressure and heart rate regulations in rats, Life Sci. 92 (2013) 1202-1207.

[14] H. Yamamoto, T. Kawada, S. Shimizu, A. Kamiya, M.J. Turner, S. Miyazaki, M. Sugimachi, Acute effects of intravenous nifedipine or azelnidipine on open-loop baroreflex static characteristics in rats, Life Sci. 126 (2015) 37-41.

[15] D.E. Mohrman, L.J. Heller, Cardiovascular Physiology, 7th edn, McGraw-Hill, New York, (2010) pp 246-250.

[16] T. Sato, T. Kawada, M. Inagaki, T. Shishido, H. Takaki, M. Sugimachi, K. Sunagawa, New analytic framework for understanding sympathetic baroreflex 
control of arterial pressure, Am. J. Physiol. 276 (1999) H2251-H2261.

[17] D.A. Sica, Centrally acting antihypertensive agents: an update, J. Clin. Hypertens. (Greenwich). 9 (2007) 399-405.

[18] A.A. Shoukas, C.A. Callahan, J.M. Lash, E.B. Haase, New technique to completely isolate carotid sinus baroreceptor regions in rats, Am. J. Physiol. 260 (1991) H300-H303.

[19] T. Sato, T. Kawada, H. Miyano, T. Shishido, M. Inagaki, R. Yoshimura, T. Tatewaki, M. Sugimachi, J. Jr. Alexander, K. Sunagawa, New simple methods for isolating baroreceptor regions of carotid sinus and aortic depressor nerves in rats, Am. J. Physiol. 276 (1999) H326-H332.

[20] B.B. Kent, J.W. Drane, B. Blumenstein, J.W. Manning, A mathematical model to assess changes in the baroreceptor reflex, Cardiology 57 (1972) 295-310.

[21] T. Kawada, T. Shishido, M. Inagaki, C. Zheng, Y. Yanagiya, K. Uemura, M. Sugimachi, K. Sunagawa, Estimation of baroreflex gain using a baroreflex equilibrium diagram, Jpn. J. Physiol. 52 (2002) 21-29.

[22] S.A. Glantz, Primer of biostatistics, 5th edn, McGraw-Hill, New York (2002).

[23] C. Vayssetts-Courchay, F. Bouysset, A.A. Cordi, M. Laubie, T.J. Verbeuren, A comparative study of the reversal by different $\alpha_{2}$-adrenoceptor antagonists of the central sympatho-inhibitory effect of clonidine, Br. J. Pharmacol. 117 (1996) $578-593$.

[24] A.F. Arnsten, J.X. Cai, P.S. Goldman-Rakic, The alpha-2 adrenergic agonist guanfacine improves memory in aged monkeys without sedative or hypotensive side effects: evidence for alpha-2 receptor subtypes, J. Neurosci. 8 (1988) 4287-4298. 
[25] S.Z. Langer, $\alpha_{2}$-Adrenoceptors in the treatment of major neuropsychiatric disorders. Trends Pharmacol. Sci. 36 (2015) 196-202.

[26] J.P. Hieble, D.C. Kolpak, Mediation of the hypotensive action of systemic clonidine in the rat by $\alpha_{2}$-adrenoceptors, Br. J. Pharmacol 110 (1993) 16351639.

[27] E. Müller-Schweinitzer, Tissue specific susceptibility of alpha-adrenoceptor mediated vasoconstriction to nifedipine, Naunyn-Schmiedeberg's Arch. Pharmacol. 324 (1983) 64-69.

[28] P.E. Hicks, I.C. Medgett, S.Z. Langer, Postsynaptic alphaz-adrenergic receptor-mediated vasoconstriction in SHR tail arteries in vitro, Hypertension 6(Suppl I) (1984) I12-I18.

[29] T. Kawada, T. Akiyama, S. Shimizu, A. Kamiya, K. Uemura, Y. Sata, M. Shirai, M. Sugimachi, Central vagal activation by alpha2-adrenergic stimulation is impaired in spontaneously hypertensive rats, Acta. Physiol. (Oxf.) 206 (2012) 72-79.

[30] J.M. Foody, M.H. Farrell, H.M. Krumholz, $\beta$-Blocker therapy in heart failure: scientific review, JAMA 287 (2002) 883-889.

[31] M.D. Flather, S. Yusuf, L. Køber, M. Pfeffer, A. Hall, G. Murray, C. Torp-Pedersen, S. Ball, J. Pogue, L. Moyé, E. Braunwald, Long-term ACE-inhibitor therapy in patients with heart failure or left-ventricular dysfunction: a systematic overview of data from individual patients, ACE-inhibitor Myocardial Infarction Collaborative Group. Lancet 355 (2000) 1575-1581.

[32] B. Pitt, P.A. Poole-Wilson, R. Segal, F.A. Martinez, K. Dickstein, A.J. Camm, M.A. Konstam, G. Riegger, G.H. Klinger, J. Neaton, D. Sharma, B. Thiyagarajan, 
Effect of losartan compared with captopril on mortality in patients with symptomatic heart failure: randomized trial—the Losartan Heart Failure Survival Study ELITE II, Lancet 355 (2000) 1582-1587.

[33] Z. Weiguo, B.S. Huang, F.H.H. Leenen, Brain renin-angiotensin system and sympathetic hyperactivity in rats after myocardial infarction. Am. J. Physiol. 276 (1999), H1608-H1615.

[34] M. Li, C. Zheng, T. Sato, T. Kawada, M. Sugimachi, K. Sunagawa, Vagal nerve stimulation markedly improves long-term survival after chronic heart failure in rats, Circulation 109 (2004) 120-124.

[35] M. Li, C. Zheng, T. Kawada, M. Inagaki, K. Uemura, T. Shishido, M. Sugimachi, Donepezil markedly improves long-term survival in rats with chronic heart failure after extensive myocardial infarction, Circ. J. 77 (2013) 2519-2525.

[36] M. Li, C. Zheng, T. Kawada, M. Inagaki, K. Uemura, M. Sugimachi, Adding the acetylcholinesterase inhibitor, donepezil, to losartan treatment markedly improves long-term survival in rats with chronic heart failure, Eur. J. Heart Fail. 16 (2014) 1056-1065.

[37] T. Kawada, T. Akiyama, S. Shimizu, A. Kamiya, K. Uemura, M.J. Turner, M. Shirai, M. Sugimachi, Sympathetic afferent stimulation inhibits central vagal activation induced by intravenous medetomidine in rats, Acta. Physiol. (Oxf.) 209 (2013) 55-61.

[38] H. Num, S.M. Clinton, N.L. Jackson, I.A. Kerman, Learned helplessness and social avoidance in the Wistar-Kyoto rat, Front. Behav. Neurosci. 8 (2014) 109. 


\section{Figure legends}

\section{Figure 1.}

Typical recordings of carotid sinus pressure (CSP), arterial pressure (AP), sympathetic nerve activity (SNA), and heart rate (HR) before and after administering guanfacine at a high dose. In the panels of AP and HR, the gray and black lines indicate $200-\mathrm{Hz}$ resampled signals and 2-s moving averaged signals, respectively. In the panels of SNA, the gray and black lines represent $10-\mathrm{Hz}$ resampled signals and 2-s moving averaged signals, respectively. The baroreflex responses in the second input cycle (S2) were treated as control data. The baroreflex responses in the fifth input cycle (S5) were used to evaluate the drug effect. Guanfacine solution (1 ml/kg) was intravenously administered as a bolus $1 \mathrm{~min}$ after the completion of the S2 cycle (down arrow).

\section{Figure 2.}

Typical recordings of carotid sinus pressure (CSP), arterial pressure (AP), sympathetic nerve activity (SNA), and heart rate (HR) before and after administering guanfacine at a low dose. In the panels of AP and HR, the gray and black lines indicate $200-\mathrm{Hz}$ resampled signals and 2-s moving averaged signals, respectively. In the panels of SNA, the gray and black lines represent $10-\mathrm{Hz}$ resampled signals and 2-s moving averaged signals, respectively. The baroreflex responses in the second input cycle (S2) were treated as control data. The baroreflex responses in the fifth input cycle (S5) were used to evaluate the drug effect. Guanfacine solution (1 ml/kg) was intravenously administered as a bolus $1 \mathrm{~min}$ after the completion of the S2 cycle (down arrow). While HR showed a notch-like response during S1 and S2, the 
phenomenon was not commonly observed across animals.

\section{Figure 3.}

Open-loop static characteristics of the baroreflex total reflex arc $(A)$, heart rate $(H R)$ regulation (B), neural arc (C), and peripheral arc (D) obtained before (open circles) and after (filled circles) administering the high dose of guanfacine $(n=7)$, and those $(\mathrm{E}-\mathrm{H})$ before (open circles) and after (gray circles) administering the low dose of guanfacine $(n=5)$. The high dose of guanfacine decreased AP at low CSP levels whereas it increased AP at high CSP levels (Fig. 2A). This phenomenon was explained by the suppression of SNA in the neural arc that was dependent on the CSP levels (Fig. 2C) and the increase in AP in the peripheral arc that was nearly independent of the SNA levels (Fig. 2D). The high dose of guanfacine decreased the maximum HR by reducing the response range and the minimum HR (Fig. $2 \mathrm{C}$ ). The low dose of guanfacine revealed weaker effects on both the neural and peripheral arcs.

\section{Figure 4.}

Baroreflex equilibrium diagrams constructed from mean parameters of the neural and peripheral arcs obtained from the high dose (A) and low dose (B) protocols of intravenous guanfacine. The intersection between the neural and peripheral arcs yields the operating point at which sympathetic nerve activity (SNA) and arterial pressure (AP) are settled under baroreflex closed-loop conditions. While the high dose of guanfacine significantly attenuated the neural arc toward lower SNA, it shifted the peripheral arc toward higher AP. As a result, the operating-point AP was not 
significantly changed by guanfacine despite the significant decrease in the operating-point SNA (the open circle versus the filled circle). The operating-point AP might have been significantly decreased had the peripheral arc remained unchanged (the open triangle). Decreasing the dose of guanfacine only contributed to a reduction in the changes in the neural and peripheral arcs (see Discussion for details). 
Table 1. Parameters of open-loop static characteristics of the carotid sinus baroreflex and heart rate (HR) regulation obtained in Protocols 1 and 2.

\begin{tabular}{|c|c|c|c|c|c|c|}
\hline & \multicolumn{3}{|c|}{ Higher dose $(n=7)$} & \multicolumn{3}{|c|}{ Lower dose $(n=5)$} \\
\hline & Control & Guanfacine & $P$ value & Control & Guanfacine & $P$ value \\
\hline \multicolumn{7}{|l|}{ Total Reflex Arc } \\
\hline$P_{1}, \mathrm{mmHg}$ & $86.8 \pm 6.4$ & $38.4 \pm 12.9 * *$ & 0.006 & $86.5 \pm 7.2$ & $78.2 \pm 6.7$ & 0.158 \\
\hline$P_{2}, \mathrm{mmHg}^{-1}$ & $0.156 \pm 0.011$ & $0.093 \pm 0.017 * *$ & 0.006 & $0.157 \pm 0.020$ & $0.142 \pm 0.028$ & 0.524 \\
\hline$P_{3}, \mathrm{mmHg}$ & $108.6 \pm 5.9$ & $106.3 \pm 4.7$ & 0.566 & $110.2 \pm 7.8$ & $102.0 \pm 4.2$ & 0.130 \\
\hline$P_{4}, \mathrm{mmHg}$ & $79.3 \pm 7.5$ & $93.2 \pm 8.7^{*}$ & 0.012 & $61.7 \pm 8.2$ & $69.0 \pm 7.4 * *$ & 0.004 \\
\hline $\mathrm{G}_{\max }, \mathrm{mmHg} / \mathrm{mmHg}$ & $3.38 \pm 0.36$ & $1.07 \pm 0.56 * *$ & 0.002 & $3.35 \pm 0.44$ & $2.70 \pm 0.44$ & 0.099 \\
\hline \multicolumn{7}{|l|}{ Heart Rate Control } \\
\hline$P_{1}, \mathrm{bpm}$ & $61.7 \pm 7.1$ & $11.7 \pm 4.2^{* *}$ & $<0.001$ & $55.1 \pm 15.4$ & $38.0 \pm 14.5 * *$ & 0.002 \\
\hline$P_{2}, \mathrm{mmHg}^{-1}$ & $0.116 \pm 0.023$ & $0.092 \pm 0.022$ & 0.290 & $0.085 \pm 0.018$ & $0.115 \pm 0.033$ & 0.131 \\
\hline$P_{3}, \mathrm{mmHg}$ & $114.6 \pm 4.5$ & $126.3 \pm 5.4$ & 0.076 & $123.8 \pm 5.9$ & $114.3 \pm 5.7$ & 0.102 \\
\hline$P_{4}, \mathrm{bpm}$ & $331.3 \pm 7.8$ & $317.4 \pm 8.3 * *$ & 0.002 & $346.0 \pm 6.5$ & $342.1 \pm 9.0$ & 0.285 \\
\hline$G_{\max }, \mathrm{bpm} / \mathrm{mmHg}$ & $1.71 \pm 0.38$ & $0.25 \pm 0.09 * *$ & 0.004 & $1.06 \pm 0.26$ & $0.91 \pm 0.34$ & 0.263 \\
\hline \multicolumn{7}{|l|}{ Neural Arc } \\
\hline$P_{1}, \%$ & $90.4 \pm 2.3$ & $33.4 \pm 10.7 * *$ & 0.003 & $87.8 \pm 2.7$ & $73.5 \pm 4.7$ & 0.068 \\
\hline$P_{2}, \mathrm{mmHg}^{-1}$ & $0.128 \pm 0.009$ & $0.140 \pm 0.017$ & 0.393 & $0.126 \pm 0.018$ & $0.139 \pm 0.015$ & 0.525 \\
\hline$P_{3}, \mathrm{mmHg}$ & $109.4 \pm 5.5$ & $102.4 \pm 3.9$ & 0.119 & $114.7 \pm 7.4$ & $106.5 \pm 5.3$ & 0.063 \\
\hline$P_{4}, \%$ & $11.4 \pm 1.9$ & $2.6 \pm 1.5^{* *}$ & 0.001 & $12.3 \pm 2.2$ & $5.7 \pm 1.4 *$ & 0.046 \\
\hline$G_{\max }, \% / m m H g$ & $2.91 \pm 0.26$ & $1.13 \pm 0.44 *$ & 0.011 & $2.80 \pm 0.48$ & $2.48 \pm 0.17$ & 0.487 \\
\hline \multicolumn{7}{|l|}{ Peripheral Arc } \\
\hline$P_{\text {slope }}, \mathrm{mmHg} / \%$ & $0.970 \pm 0.054$ & $1.223 \pm 0.175$ & 0.110 & $0.997 \pm 0.088$ & $1.026 \pm 0.047$ & 0.554 \\
\hline$P_{\text {intercept }}, \mathrm{mmHg}$ & $67.6 \pm 7.1$ & $92.8 \pm 8.5^{* *}$ & $<0.001$ & $46.3 \pm 9.7$ & $61.4 \pm 7.3^{*}$ & 0.012 \\
\hline \multicolumn{7}{|l|}{ Operating Point } \\
\hline AP, $\mathrm{mmHg}$ & $114.6 \pm 4.3$ & $108.9 \pm 7.2$ & 0.311 & $111.2 \pm 6.9$ & $105.9 \pm 5.4$ & 0.126 \\
\hline SNA, \% & $47.4 \pm 5.9$ & $13.8 \pm 4.0 * *$ & $<0.001$ & $64.5 \pm 5.7$ & $43.0 \pm 5.1 * *$ & 0.004 \\
\hline Gain, $\mathrm{mmHg} / \mathrm{mmHg}$ & $2.53 \pm 0.42$ & $1.09 \pm 0.57 *$ & 0.022 & $2.55 \pm 0.48$ & $2.41 \pm 0.18$ & 0.706 \\
\hline
\end{tabular}

Data are presented as means $\pm \mathrm{SE}$. ${ }^{* *} \mathrm{P}<0.01$ and ${ }^{*} \mathrm{P}<0.05$ by paired t-test. $P_{1}$ to $P_{4}$ : parameters of a 4-parameter logistic function, $P_{1}$ : response range $P_{2}$ : slope coefficient, $P_{3}$ : midpoint input pressure, $P_{4}$ : minimum value, $G_{\text {max }}$ : maximum gain or maximum slope calculated from $P_{1 \times} P_{2} / 4, P_{\text {slope }}$ : slope of linear regression, $P_{\text {intercept }}$ intercept of linear regression, AP: arterial pressure, SNA: sympathetic nerve activity, bpm: beats/min. The operating-point was determined by equilibrating the neural and peripheral arc models on a pressure-SNA plane. 
Figure 1
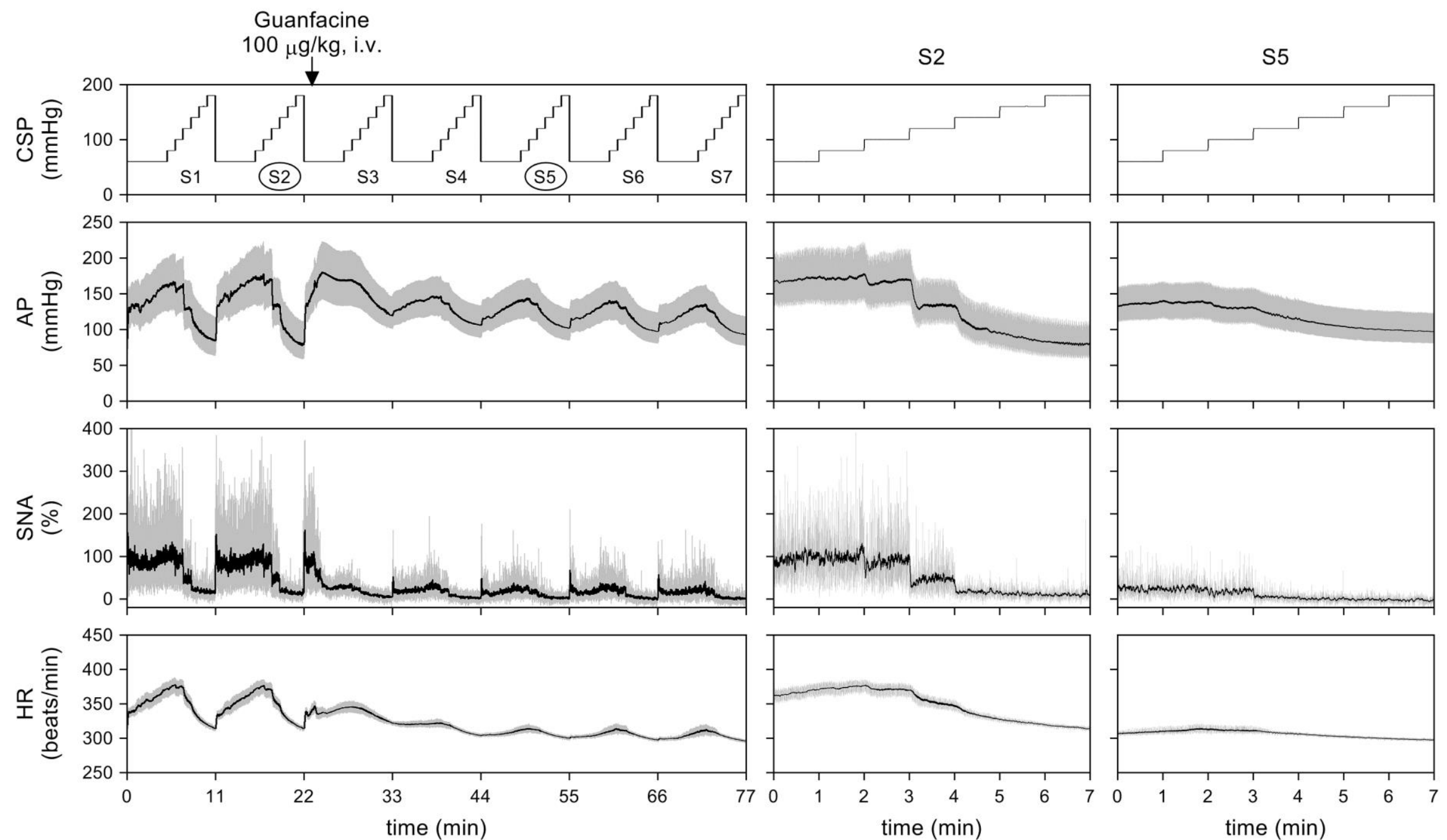
Figure 2

Guanfacine

$20 \mu \mathrm{g} / \mathrm{kg}$, i.v.
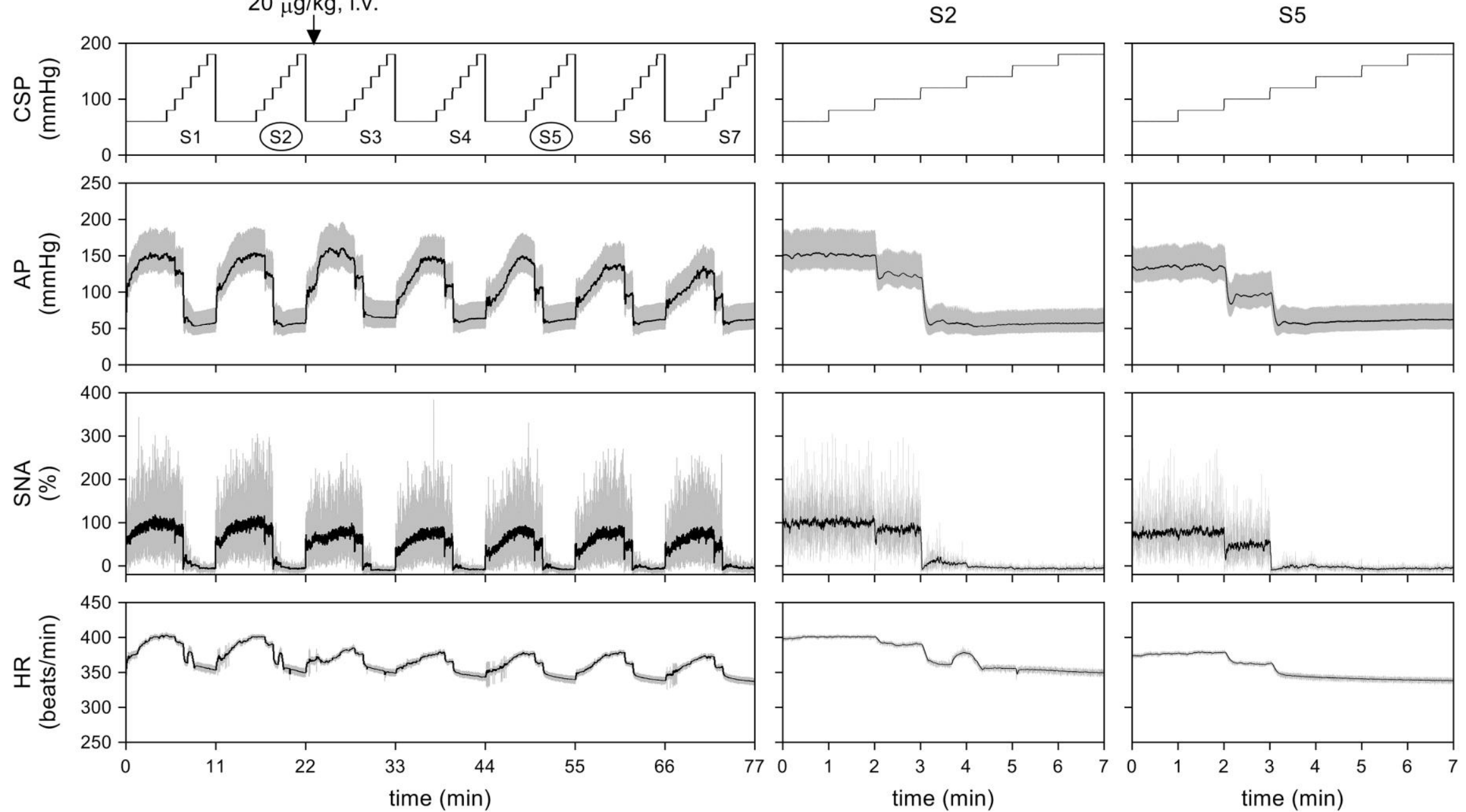
Figure 3
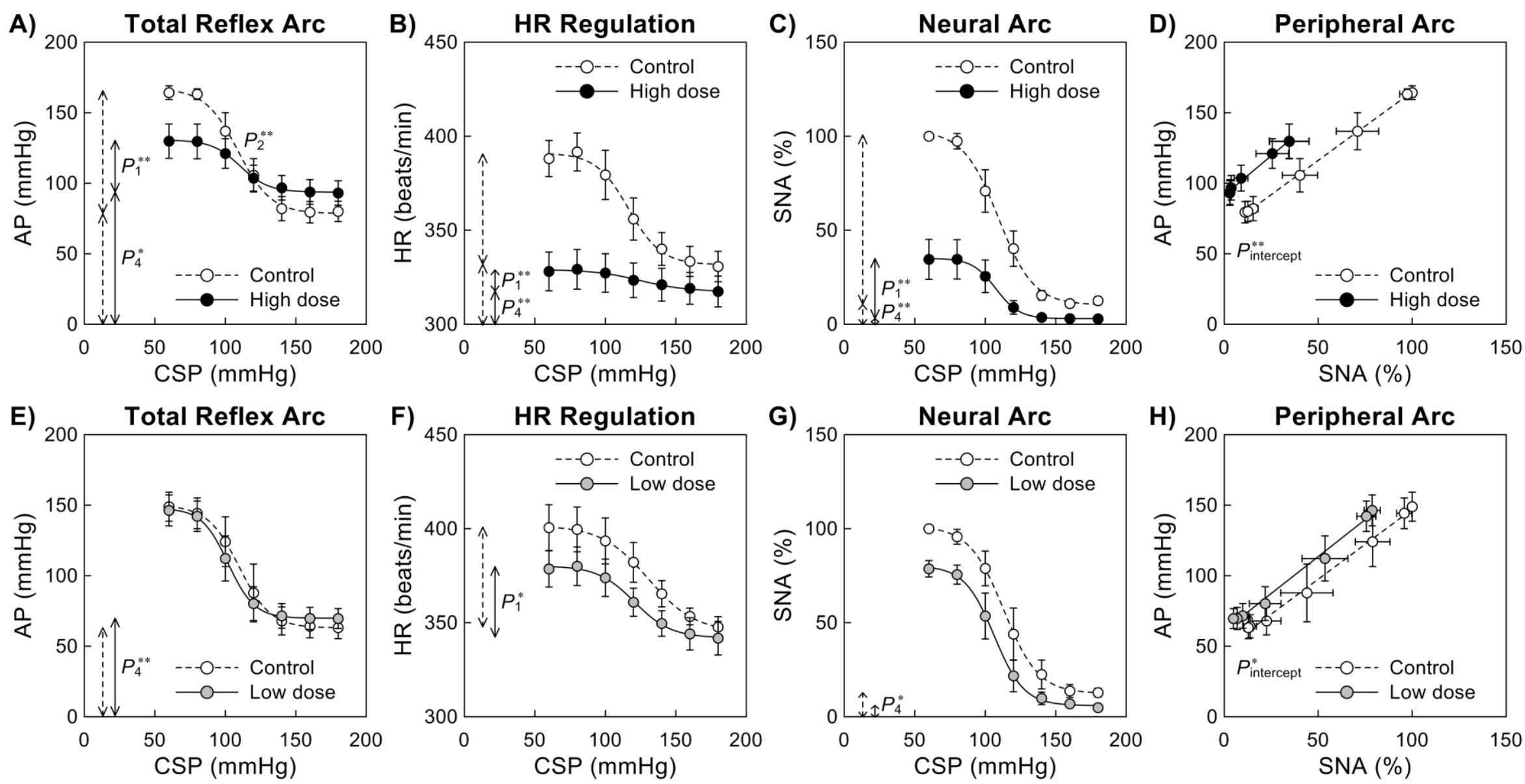
Figure 4
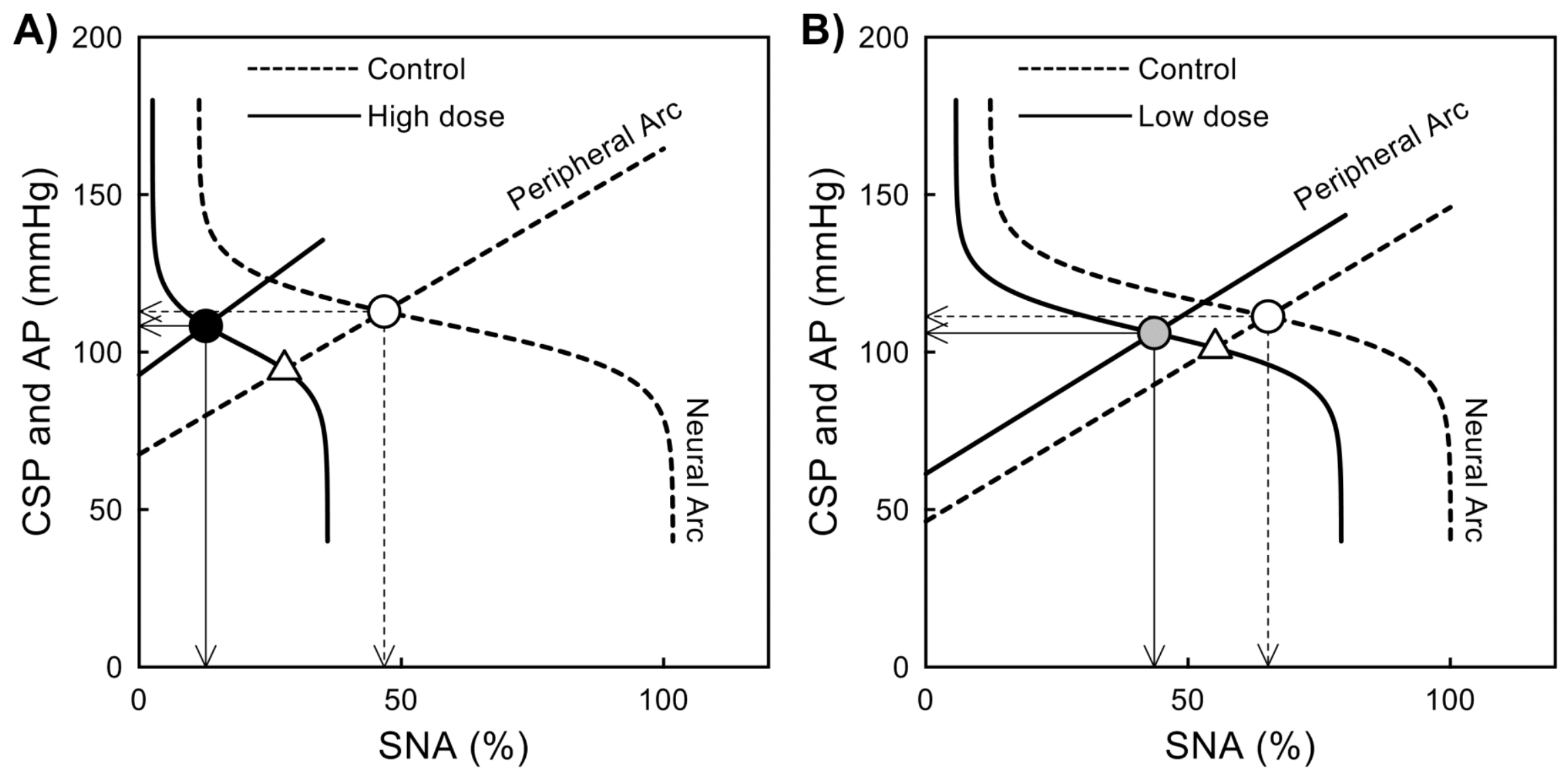